\title{
Ligadura interesfintérica de trayecto fistuloso como tratamiento de fístula anorrectal compleja
}

\author{
Ligation of intersphinteric fistula tract as \\ treatment of complex anorectal fistula \\ Karla Ivonne Luján-Mendoza, ${ }^{*}$ Agustín Güemes-Quinto, ${ }^{*}$ Carlos Cosme-Reyes ${ }^{\ddagger}$
}

\begin{abstract}
Palabras clave:
Fístula anal, fístula anal compleja, fístula

transesfintérica

alta, ligadura

interesfintérica del trayecto fistuloso, incontinencia fecal.
\end{abstract}

Keywords:

Fistula-in-ano, complex anal fistula, high transsphinteric

fistula, ligation of intersphincteric fistula tract, fecal incontinence.

* Médico Residente.

¥ Médico adscrito.

Servicio de Coloproctología del Hospital General de México "Dr.

Eduardo Liceaga".

Ciudad de México.

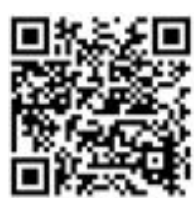

\section{RESUMEN}

Objetivo: Demostrar que uno de los tratamientos efectivos para la resolución de fístulas anales complejas transesfintéricas altas es la técnica de ligadura del trayecto interesfintérico, ya que es uno de los procedimientos que ha demostrado adecuados resultados para la resolución de la fístula, así como la preservación del esfínter anal y la continencia. Material y método: Se realizó un estudio prospectivo y observacional de pacientes intervenidos quirúrgicamente con la técnica de ligadura del trayecto interesfintérico por fístula anal compleja (transesfintérica alta), de origen criptoglandular durante el periodo comprendido entre enero del 2016 y agosto del 2018 en el Servicio de Coloproctología del Hospital General de México “Dr. Eduardo Liceaga” de la Ciudad de México. Se analizaron las características clínicas, la técnica quirúrgica y su resultado. Resultados: Fueron incluidos un total de 23 pacientes con fístula transesfintérica alta, a los cuales se les realizó técnica de ligadura del trayecto interesfintérico. Sólo uno de los pacientes presentó recurrencia de la fístula. En el seguimiento postoperatorio, un mes después de la cirugía, tres pacientes presentaron absceso perianal en el sitio de la incisión (13.04\%). Ningún paciente presentó incontinencia fecal posterior a procedimiento quirúrgico, la mediana del score de Wexner fue 0. Conclusión: En nuestra experiencia, la técnica de ligadura del trayecto interesfintérico resultó una cirugía segura, reproducible, con escasa morbilidad y nula incontinencia fecal, con un porcentaje de éxito alrededor de $90 \%$, por lo que podría considerarse la primera línea de tratamiento quirúrgico en fístula anal compleja transesfintérica alta.

\section{ABSTRACT}

Objective: To demonstrate that one of the effective treatments for the resolution of high transsphincteric complex anal fistulas is the ligature of intersphinteric tract technique, since it is one of the procedures that has demonstrated adequate results for the resolution of the fistula, as well as the preservation of the anal sphincter and the continence. Material and method: This is a prospective and observational study of patients surgically treated with the ligature of intersphinteric tract technique for complex anal fistula (high transsphinteric), of cryptoglandular origin during the period between January 2016 and August 2018 in the Coloproctology Unit at the Hospital General de México "Dr. Eduardo Liceaga" in Mexico City. The clinical characteristics, the surgical technique and its result were analyzed. Results: A total of 23 patients with high transsphinteric fistula were included, who underwent the ligature of intersphinteric tract technique. only in one, recurrence of the fistula occurred. In the postoperative follow-up, one month after surgery, three patients presented with a perianal abscess at the site of the incision (13.04\%), which required drainage and subsequent management with antibiotic. No patient presented fecal incontinence after surgical procedure, the median Wexner score was 0. Conclusion: In our experience, the ligature of intersphinteric tract technique was a safe, reproducible surgery with low morbidity and no fecal incontinence, with a success rate of around $90 \%$, and could be considered as the first line of treatment in high trans-sphincteric complex anal fistula.

\section{INTRODUCCIÓN}

$\mathrm{D}$ espués de someterse al drenaje de un absceso anorrectal, aproximadamente una tercera parte de los pacientes desarro- llarán una fístula anal, la cual es una conexión anormal entre el conducto anal y la piel perianal. Las fístulas se manifiestan con dolor, inflamación y drenaje purulento de forma persistente o intermitente. El origen

Citar como: Luján-Mendoza KI, Güemes-Quinto A, Cosme-Reyes C. Ligadura interesfintérica de trayecto fistuloso como tratamiento de fístula anorrectal compleja. Cir Gen. 2020; 42(1): 13-18. doi: 10.35366/92706 
más común de estas fístulas es la infección criptoglandular. ${ }^{1-3}$

Las fístulas se clasifican en simples o complejas, estas últimas incluyen trayectos transesfintéricos altos que involucran más de 30\% del esfínter anal, fístulas supraesfintéricas, extraesfintéricas, fístulas recurrentes, fístulas con múltiples trayectos y todas las fístulas de localización anterior en las mujeres, entre otras. ${ }^{4-6}$

Los objetivos fundamentales en el tratamiento de la fístula anal son erradicar la sepsis, cerrar el trayecto fistuloso, evitar su recurrencia y, lo más importante, preservar la continencia. ${ }^{7}$

Una de estas técnicas preservadoras del esfínter anal para el tratamiento de fístulas complejas es la ligadura del trayecto interesfintérico (LIFT por sus siglas en inglés), que fue descrita por primera vez por Rojanasakul y colaboradores en el año 2007, principalmente

\section{Tabla 1: Características clínicas de la serie.}

\begin{tabular}{lc} 
Variable & N $=\mathbf{2 3}$ \\
\hline Edad (años) & $23-70$ \\
Sexo & \\
$\quad$ Masculino & 20 \\
Femenino & 3 \\
Tabaquismo & \\
$\quad$ Positivo & 1 \\
$\quad$ Negativo & 22 \\
Diabetes mellitus & \\
$\quad$ Positivo & 1 \\
$\quad$ Negativo & 22 \\
Hipertensión arterial & \\
$\quad$ Positivo & 2 \\
$\quad$ Negativo & 21 \\
Obesidad (IMC) & \\
$\quad>25$ & 7 \\
$\quad$ <25 & 16 \\
Tiempo de evolución (meses) & $4-24$ \\
Cirugía previa & \\
Sí & 10 \\
No & 13 \\
Setón previo & \\
Sí & \\
No & 14 \\
\hline
\end{tabular}

IMC = índice de masa corporal.

\begin{tabular}{l} 
Tabla 2: Características de las \\
fístulas y técnica quirúrgica. \\
\multicolumn{2}{|c}{$\mathbf{N}=\mathbf{2 3}$} \\
\hline Variable \\
\hline Tipo de fístula \\
$\quad$ Transesfintérica alta \\
Localización \\
$\quad$ Anterior \\
$\quad$ Posterior \\
Técnica LIFT \\
$\quad$ Sólo ligadura \\
Trayecto \\
$<3 \mathrm{~cm}$ \\
$>3 \mathrm{~cm}$ \\
\end{tabular}

para el tratamiento de las fístulas transesfintéricas altas..$^{8-12}$

Estos autores plantearon el cierre del orificio primario (interno) para evitar que las partículas de materia fecal entraran en el trayecto de la fístula y la ligadura del trayecto interesfintérico, para así eliminar el foco séptico. Las contraindicaciones para realizar esta técnica incluyen abscesos anorrectales asociados al trayecto, enfermedad inflamatoria intestinal activa y fístulas relacionadas a malignidad. Múltiples reportes en la literatura reportan una tasa de éxito que va del 47 al 95\%.13-17

Algunos autores consideran que se debe colocar un setón de drenaje ocho semanas previas a la realización de un LIFT, ya que consideran que esta medida elimina el foco séptico y promueve la fibrosis del trayecto fistuloso, facilitando posteriormente la disección del espacio interesfintérico y la ligadura del trayecto. ${ }^{18-25}$

El objetivo de este trabajo es describir la experiencia con técnica de LIFT en fístulas anales transesfintéricas altas (complejas) de un mismo grupo quirúrgico, y describir las modificaciones que hemos realizado a esta técnica.

\section{MATERIAL Y MÉTODO}

Se realizó un estudio prospectivo, observacional y longitudinal de pacientes intervenidos quirúrgicamente con técnica de LIFT por fístula anal compleja (transesfintérica alta), de origen 


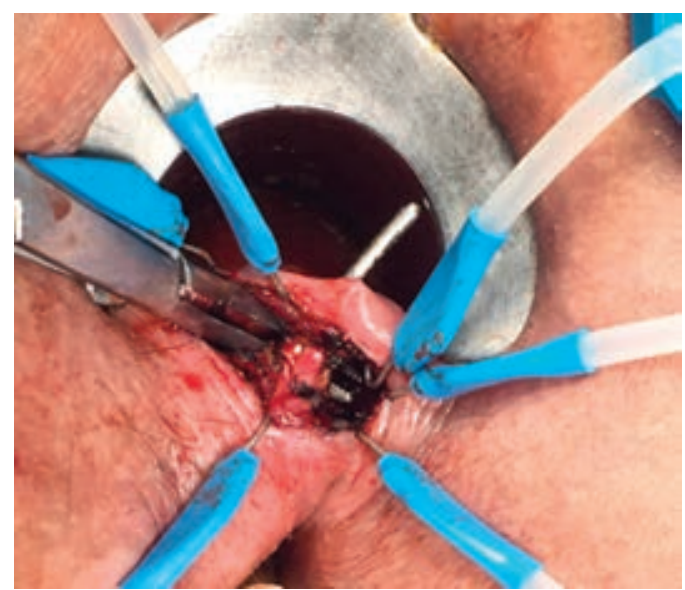

Figura 1: Trayecto fistuloso disecado.

criptoglandular durante el periodo comprendido entre enero del 2016 y agosto del 2018, en el Servicio de Coloproctología del Hospital General de México "Dr. Eduardo Liceaga" de la Ciudad de México, por un mismo grupo quirúrgico. La media de seguimiento postoperatorio de los pacientes fue de 12 meses. Se consideraron pacientes con fístulas transesfintéricas altas, evidenciadas clínicamente y confirmadas mediante la realización de un ultrasonido endoanal (Tablas 1 y 2).

Los criterios de inclusión fueron los siguientes: fistula anal compleja de origen criptoglandular con trayecto transesofintérico alto, con o sin cirugías previas, con o sin colocación de setón de drenaje, y con un orificio primario único. En todos los pacientes se utilizó profilaxis antibiótica con metronidazol 500 mg en dosis única una hora previa a procedimiento quirúrgico. Todos los pacientes fueron intervenidos con bloqueo mixto y en posición de navaja sevillana.

Los pasos fundamentales del procedimiento son: tacto rectal y anoscopia completa, identificación y canalización mediante un estilete del trayecto fistuloso, previo retiro del setón en los casos en los que se presentaba, incisión de aproximadamente $2 \mathrm{~cm}$ transversal en el espacio interesfintérico, disección hasta lograr identificar el trayecto fistuloso, el cual se diseca por completo y se refiere en sus dos extremos con seda 2-0, se retira el estilete y se legra el trayecto fistuloso, se colocan dos puntos trans- fictivos con Vicryl ${ }^{\circledR}$ 2-0 en cada extremo, es decir, lo más próximo al esfínter anal interno y al esfínter anal externo (Figura 1).

Se ligan ambas referencias de seda y se secciona trayecto fistuloso con bisturí, se inyecta $1 \mathrm{ml}$ de peróxido de hidrógeno a través del orificio secundario, asegurándonos que no exista fuga hacia el espacio interesfintérico. En los casos en los que se presentó fuga se realizó una nueva ligadura con Vicryl ${ }^{\circledR}$ 2-0 y se verificó nuevamente con peróxido de hidrógeno. Se afrontó la herida quirúrgica con puntos simples de Vicryl ${ }^{\circledR} 3-0$, dejando suficiente espacio en el centro de la herida para favorecer el drenaje (Figura 2).

La técnica original recomienda disecar completamente el trayecto fistuloso; sin embargo, nosotros consideramos que es mejor no adelgazar el trayecto, ya que se previene el desgarro de éste.

El postoperatorio se manejó con analgésicos intravenosos como ketorolaco $30 \mathrm{mg}$ cada 12 horas, paracetamol $500 \mathrm{mg}$ cada ocho horas y metronidazol intravenoso $500 \mathrm{mg}$ cada ocho horas, lo cual se continuó con vía oral durante 10 días. Todos los pacientes fueron egresados del hospital a las 24 horas de haber realizado el procedimiento quirúrgico. Se realizó la evaluación postoperatoria en consulta externa a los

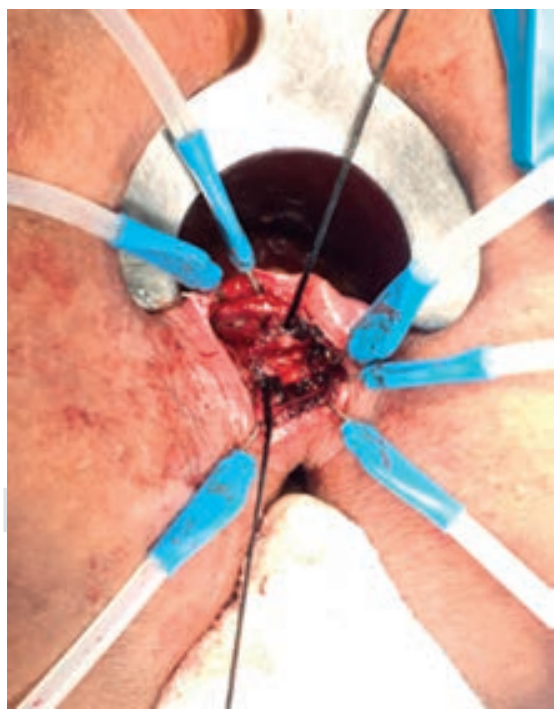

Figura 2: Trayecto fistuloso referido con seda y seccionado. 
10 días, 30 días, tres meses, seis meses y a un año del procedimiento quirúrgico, en algunos pacientes hasta 24 meses. El seguimiento consistió únicamente en evaluación clínica.

Se definió como curación el cierre de la herida y del orificio fistuloso secundario, así como la ausencia de síntomas. La continencia se valoró mediante la escala de Wexner.

\section{RESULTADOS}

Durante el periodo de estudio fueron incluidos 23 pacientes, 20 hombres y tres mujeres. La mediana de edad fue de 46 años, con predominio de los hombres (87\%). Sólo tres de los pacientes presentaban comorbilidades como diabetes tipo 2 e hipertensión arterial sistémica (13.04\%). Uno de ellos con antecedente de tabaquismo. Siete de los pacientes presentaban obesidad (30.4\%), nueve contaban con setón de drenaje (39.13\%); sin embargo, esto no significó ninguna ventaja para la realización posterior de LIFT. Todos los sujetos presentaban fístulas transesfintéricas altas, $82.6 \%$ con un trayecto fistuloso mayor de $3 \mathrm{~cm}$, el de mayor longitud fue de $8 \mathrm{~cm}$. La longitud del trayecto fistuloso no influyó en el resultado. Se realizó LIFT en todos los pacientes y uno de ellos $(4.3 \%)$ presentó recurrencia a los nueve meses y una fístula transesfintérica baja anterior. El promedio de seguimiento fue de 12 meses, siendo el menor de ocho meses y el mayor de 24 meses, ningún paciente se perdió en el seguimiento. A pesar de ser consideradas fístulas complejas, sólo se evidenció recurrencia en un paciente el cual se programó para fistulotomía, con resolución del cuadro.

En el seguimiento postoperatorio, tres pacientes presentaron absceso perianal un mes después (13.04\%), que ameritó drenaje de éste con manejo antibiótico a base de metronidazol 500 mg vía oral cada ocho horas durante 10 días, y posteriormente presentaron resolución del cuadro. Cuatro pacientes (17.39\%) presentaron salida de líquido seropurulento no fétido por orificio secundario, se manejaron con metronidazol $500 \mathrm{mg}$ vía oral cada ocho horas durante 10 días con posterior resolución del cuadro. Las heridas que presentaron salida de líquido seroso o serohemático posterior al procedimiento quirúrgico cerraron entre
6-8 semanas, en algunos casos se administró tratamiento antibiótico con metronidazol 500 mg vía oral cada ocho horas durante 10 días. Ningún individuo presentó incontinencia fecal después del procedimiento quirúrgico, la mediana del score de Wexner fue de 0.

\section{DISCUSIÓN}

El manejo de la fístula anal es un desafío para el cirujano colorrectal, los resultados en la literatura son variables. El manejo quirúrgico ideal debe incluir cierre de la fístula, baja tasa de recurrencia y la preservación del esfínter anal.

El concepto de abordaje interesfintérico fue publicado por primera vez por el grupo de St. Marks en 1993, con una serie de 13 pacientes con drenaje del espacio interesfintérico, cierre de los orificios fistulosos y cierre de la herida. Sin embargo, la técnica LIFT, preservadora de la continencia fecal en fístulas complejas, fue descrita por primera vez en 2007 por Rojanasakul y colaboradores para las fístulas anales complejas como técnica preservadora de esfínteres, con una tasa de éxito que va de $40-95 \%$. De acuerdo con lo reportado en la literatura, esta técnica se puede realizar en fístulas interesfintéricas o transesfintéricas bajas; sin embargo, se han reportado altas tasas de recidiva y se considera que en la mayor parte de estos casos se puede realizar una fistulotomía. Sólo un paciente de nuestra serie reportó recurrencia como fístula simple y se resolvió con realización de fistulotomía.

El impacto sobre la continencia suele ser escaso o nulo, como en nuestra serie de casos, por lo que representa un punto importante de la técnica, ya que esto no sucede en todas las técnicas para tratar las fístulas anales complejas.

En nuestra experiencia, la colocación de setón de drenaje, previo a la realización de LIFT, no ofrece ninguna ventaja, ya que los pacientes que no presentaron colocación previa de dicho setón presentaron los mismos resultados a corto y mediano plazo. Es sabido que la presencia de un trayecto poco fibroso dificulta o impide la realización de esta técnica, sin embargo, la colocación previa del setón no garantiza un trayecto mucho más fibroso.

La técnica LIFT constituye una muy buena opción para la resolución de fístulas complejas, 
en nuestro caso con una tasa de éxito mayor a 90\%, tomando en cuenta la adecuada selección de los pacientes y que las fístulas sean de origen criptoglandular con un trayecto transesfintérico alto. En cuanto a la técnica quirúrgica, consideramos que el paso fundamental para no presentar recidiva de la fístula es no adelgazar de forma importante el trayecto fistuloso, así como verificar que no haya fuga con la irrigación del peróxido de hidrógeno. En caso de presentarse, recomendamos el dar puntos de reforzamiento con Vicryl ${ }^{\circledR}$.

\section{CONCLUSIONES}

En nuestra experiencia, la técnica LIFT resultó una cirugía segura, reproducible, con escasa morbilidad y nula incontinencia fecal, con un porcentaje de éxito de 95\%, por lo que podría considerarse la primera línea de tratamiento quirúrgico en fístula anal compleja transesfintérica alta.

Se puede usar después de la colocación de setón o la realización de algún otro procedimiento quirúrgico que no logró la resolución de la fístula anal. Incluso si llegara a fallar la técnica LIFT, la fístula se puede tratar con fistulotomía o con nuevo procedimiento de LIFT.

\section{REFERENCIAS}

1. Lange EO, Ferrari L, Krane M, Fichera A. Ligation of intersphincteric fistula tract: a sphincter-sparing option for complex fistula-in-ano. J Gastrointest Surg. 2016; 20: 439-444.

2. Hong KD, Kang S, Kalaskar S, Wexner SD. Ligation of intersphincteric fistula tract (LIFT) to treat anal fistula: systematic review and meta-analysis. Tech Coloproctol. 2014; 18: 685-691.

3. Khadia M, Muduli IC, Das SK, Mallick SN, Bag L, Pati MR. Management of fistula-in-ano with special reference to ligation of intersphincteric fistula tract. Niger J Surg. 2016; 22: 1-4.

4. Malakorn S, Sammour T, Khomvilai S, Chowchankit I, Gunarasa S, Kanjanasilp P, et al. Ligation of intersphincteric fistula tract for fistula in ano: lessons learned from a decade of experience. Dis Colon Rectum. 2017; 60: 1065-1070.

5. Han JG, Yi BQ, Wang ZJ, Zheng Y, Cui JJ, Yu XQ, et al. Ligation of the intersphincteric fistula tract plus a bioprosthetic anal fistula plug (LIFT-Plug): a new technique for fistula-in-ano. Colorectal Dis. 2013; 15: 582-586.

6. Sirikurnpiboon S, Awapittaya B, Jivapaisarnpong P. Ligation of intersphincteric fistula tract and its modification: Results from treatment of complex fistula. World J Gastrointest Surg. 2013; 5: 123128.

7. Parthasarathi R, Gomes RM, Rajapandian S, Sathiamurthy R, Praveenraj P, Senthilnathan P, et al. Ligation of the intersphincteric fistula tract for the treatment of fistula-in-ano: experience of a tertiary care centre in South India. Colorectal Dis. 2016; 18: 496-502.

8. Tan KK, Tan IJ, Lim FS, Koh DC, Tsang CB. The anatomy of failures following the ligation of intersphincteric tract technique for anal fistula: a review of 93 patients over 4 years. Dis Colon Rectum. 2011; 54: 1368-1372.

9. Abcarian AM, Estrada JJ, Park J, Corning C, Chaudhry V, Cintron J, et al. Ligation of intersphincteric fistula tract: early results of a pilot study. Dis Colon Rectum. 2012; 55: 778-782.

10. Araújo SEA, Marcante MT, Mendes CRS, Bertoncini AB, Seid VE, Horcel LA, et al. Interesfincterial ligation of fistula tract (lift) for patients with anal fistulas: a brazilian bi-institutional experience. ABCD Arq Bras Cir Dig. 2017; 30: 235-238.

11. Galán C, López C. Patrones de recurrencia/persistencia en la operación de LIFT para la fístula anal de origen criptoglandular. Estudio observacional a largo plazo. Cir Esp. 2017; 95: 385-390.

12. Sileri P, Giarratano G, Franceschilli L, Limura E, Perrone F, Stazi A, et al. Ligation of the intersphincteric fistula tract (LIFT): a minimally invasive procedure for complex anal fistula: two-year results of a prospective multicentric study. Surg Innov. 2014; 21: 476-480.

13. Rojanasakul A, Pattanaarun J, Sahakitrungruang C, Tantiphlachiva K. Total anal sphincter saving technique for fistula-in-ano; the ligation of intersphincteric fistula tract. J Med Assoc Thai. 2007; 90: 581-586.

14. Bleier JIS, Moloo H, Goldberg SM. Ligation of the intersphincteric fistula tract: an effective new technique for com- plex fistulas. Dis Colon Rectum. 2010; 53: 43-46.

15. Shanwani A, Nor AM, Amri N. Ligation of the intersphincteric fistula tract (LIFT): a sphincter-saving technique for fistula-in-ano. Dis Colon Rectum. 2010; 53: 39-42.

16. Van Onkelen RS, Gosselink MP, Schouten WR. Ligation of the intersphincteric fistula tract in low transsphincteric fistulae: a new technique to avoid fistulotomy. Colorectal Dis. 2013; 15: 587-591.

17. Lau YC, Brown KGM, Cheong J, Byrne C. LIFT and BioLIFT: a 10-year single-centre experience of treating complex fistula-in-ano with ligation of intersphincteric fistula tract procedure with or without bio-prosthetic reinforcement (BioLIFT). J Gastrointest Surg. 2019. doi: 10.1007/s11605-019-04305-x. [Epub ahead of print]

18. Stellingwerf ME, Van Praag EM, Tozer PJ. Systematic review and meta-analysis of endorectal advancement flap and ligation of the intersphincteric fistula tract for cryptoglandular and Crohn's high perianal fistulas. BJS Open. 2019; 3: 231-241.

19. Qiu JM, Yang GG, Wang HT. Feasibility of ambulatory surgery for anal fistula with LIFT procedure. BMC Gastroenterol. 2019; 19: 81.

20. Banasiewicz T, Eder P. Statement of the expert group on the current practice and prospects for the treatment of complex perirectal fistulas in the course of Crohn's disease. Pol Przegl Chir. 2019; 91: 38-46. 
21. Osterkamp J, Gocht-Jensen P. Long-term outcomes in patients after ligation of the intersphincteric fistula tract. Dan Med J. 2019; 66: 64-71.

22. Romaniszyn M, Walega PJ. Are two better than one? VALIFT: video-assisted ligation of the intersphincteric fistula tract-a combination of two minimally invasive techniques for treatment of transsphincteric perianal fistulas. Tech Coloproctol. 2019; 23: 273-276.

23. Wen K, Gu YF, Sun XL. Long-term outcomes of ligation $\mathrm{f}$ intersphinteric fistula tract for complex fistula-in-ano: modified operative procedure experience. Arq Bras Cir Dig. 2018; 31: e1404.

24. Kang WH, Yang HK. High ligation of the anal fistula tract by lateral approach: A prospective cohort study on a modification of the ligation of the intersphincteric fistula tract (LIFT) technique. Int J Surg. 2018; 60: 9-14

25. Elfeki H, Duelund-Jakobsen J, Lundby L. Ligation of intersphincteric fistula tract procedure for the treatment of fistula in ano - a video vignette. Colorectal Dis. 2018; 20: 1154.

Consideraciones y responsabilidad ética: Privacidad de los datos. De acuerdo a los protocolos establecidos en el centro de trabajo de los autores, estos declaran que han seguido los protocolos sobre la privacidad de datos de pacientes preservado su anonimato. El consentimiento informado del paciente referido en el artículo se encuentra en poder del autor.

Financiamiento: No se recibió apoyo financiero para la realización de este trabajo.

Conflicto de intereses: Los autores declaran que no existe ningún conflicto de intereses en la realización del trabajo.

Correspondencia:

Karla Ivonne Luján-Mendoza

Esperanza Núm. 959, Int. 104,

Col. Narvarte, 03020,

Alcaldía Benito Juárez, CDMX.

E-mail: kilm_rm@hotmail.com 